\title{
Real Zeros of a Class of Hyperbolic Polynomials with Random Coefficients
}

\author{
Mina Ketan Mahanti, ${ }^{1}$ Amandeep Singh, ${ }^{2}$ and Lokanath Sahoo ${ }^{3}$ \\ ${ }^{1}$ Department of Mathematics, College of Basic Science and Humanities, OUAT, Bhubaneswar, India \\ ${ }^{2}$ DPS Kalinga, Bhubaneswar, India \\ ${ }^{3}$ Gopabandhu Science College, Athagad, India
}

Correspondence should be addressed to Mina Ketan Mahanti; minaketan_mahanti@yahoo.com

Received 7 January 2015; Revised 21 May 2015; Accepted 27 May 2015

Academic Editor: Niansheng Tang

Copyright (C) 2015 Mina Ketan Mahanti et al. This is an open access article distributed under the Creative Commons Attribution License, which permits unrestricted use, distribution, and reproduction in any medium, provided the original work is properly cited.

We have proved here that the expected number of real zeros of a random hyperbolic polynomial of the form $y=P_{n}(t)=$ $\sqrt{\left(\begin{array}{c}n \\ 1\end{array}\right)} a_{1} \cosh t+\sqrt{\left(\begin{array}{c}n \\ 2\end{array}\right)} a_{2} \cosh 2 t+\cdots+\sqrt{\left(\begin{array}{c}n \\ n\end{array}\right)} a_{n} \cosh n t$, where $a_{1}, \ldots, a_{n}$ is a sequence of standard Gaussian random variables, is $\sqrt{n} / 2+o_{p}(1)$. It is shown that the asymptotic value of expected number of times the polynomial crosses the level $y=K$ is also $\sqrt{n} / 2$ as long as $K$ does not exceed $\sqrt{2^{n} e^{\mu(n)}}$, where $\mu(n)=o(n)$. The number of oscillations of $P_{n}(t)$ about $y=K$ will be less than $\sqrt{n} / 2$ asymptotically only if $K=\sqrt{2^{n} e^{\mu(n)}}$, where $\mu(n)=O(n)$ or $n^{-1} \mu(n) \rightarrow \infty$. In the former case the number of oscillations continues to be a fraction of $\sqrt{n}$ and decreases with the increase in value of $\mu(n)$. In the latter case, the number of oscillations reduces to $o_{p}(\sqrt{n})$ and almost no trace of the curve is expected to be present above the level $y=K$ if $\mu(n) /(n \log n) \rightarrow \infty$.

\section{Introduction}

Let $(\Omega, A, \operatorname{Pr})$ be a fixed probability space and let $\left\{a_{k}(\omega)\right\}_{k=1}^{j=n}$ be a sequence of independent random variables defined on $\Omega$. The sum $a_{0}(\omega) f_{1}(t)+a_{2}(\omega) f_{2}(t)+\cdots+a_{n}(\omega) f_{n}(t)$ is traditionally known as a random algebraic polynomial if $f_{i}(t)=t^{i}$, a random trigonometric polynomial if $f_{i}(t)=$ $\cos (i t)$ or $\sin (i t)$, and a random hyperbolic polynomial if $f_{i}(t)=\cosh (i t)$ or $\sinh (i t)$. One can have useful information about the behaviour of these ensembles of polynomials if the average number of times these polynomials oscillate about the line $y=K$ is known. The reader is referred to the book by Farahmand [1] where an exhaustive account of progress made in study of random polynomials has been presented. It is to be noted that there is significantly more published literature on random algebraic and random trigonometric polynomials than that of random hyperbolic polynomials. Let

$$
y=Q_{n}(t)=\sum_{k=1}^{n} a_{k}(\omega) \cosh k t,
$$

where $a_{k}(\omega)$ are normally distributed random variables with mean zero and variance one. One knows that Das [2] first calculated the expected number of real zeros of $Q_{n}(t)$. Farahmand [3] calculated the asymptotic estimate of oscillations of $Q_{n}(t)$ about $y=K$ if $K=o(\sqrt{n})$. Some of the other works in this direction are due to Mahanti [4-6]. Wilkins [7] determined real zeros of $Q_{n}(t)$ when $\operatorname{var}\left(a_{k}(\omega)\right)=k^{p}, p \geq 0$. We observe that the asymptotic value of the oscillations of random hyperbolic polynomials is $(2 / \pi) \log n$ in each of these cases.

One is tempted to ask whether $Q_{n}(t)$ has more than $(2 / \pi) \log n$ oscillations under certain conditions. In this context, we are reminded of a recent work of Edelman and Kostlan [8] where it has been found out that the expected number of real zeros of random algebraic polynomials increases significantly if the variance of the coefficients changes from unity to $\sqrt{\left(\begin{array}{l}n \\ k\end{array}\right)}$. Therefore, we examine what effect this new assumption on variance of the coefficients has on number of 
oscillations of $Q_{n}(t)$. In other words, we calculate the number of oscillations of the polynomial

$$
y=P_{n}(t)=\sum_{k=1}^{n} a_{k}(\omega) \sqrt{\left(\begin{array}{l}
n \\
k
\end{array}\right)} \cosh k t,
$$

where $a_{k}(\omega)$ are normally distributed random variables with mean zero and variance one.

In Theorem 1 we have shown that the number of real zeros of $P_{n}(t)$ is substantially larger than that of $Q_{n}(t)$. Moreover, there is a significant difference in the way real zeros of $P_{n}(t)$ and $Q_{n}(t)$ lie on the $t$-axis. Most of the real zeros of $Q_{n}(t)$ are confined to the interval $[-1,1]$ and there are negligible numbers of them if $|t|>1$ (see [4]). But there are large numbers of real zeros of $P_{n}(t)$ outside $[-1,1]$. This phenomenon can be deduced from the formula given in (26) and Lemma 10. In fact, the number of real zeros of $P_{n}(t)$ in the region $|t|>\alpha, \alpha>1 / \sqrt{n}$, is dependent on $\alpha$. The real zeros decrease in number with increase in $\alpha$ in the region $|t|>\alpha$ and there are negligible numbers of them only when $\alpha \rightarrow \infty$.

Let $\mu(n)$ be any function of $n$ such that $\mu(n) / n \rightarrow 0$. In Theorem 2 we have shown that the number of oscillations of $P_{n}(t)$ about the line $y=K$, where $K \leq \sqrt{2^{n} e^{\mu(n)}}$, is equal to its axis crossings. Thus, for all these values of $K$ one can say that most of the oscillations of $P_{n}(t)$ that cross the $t$-axis reach up to the level $y=K$. If $\mu(n)=O(n)$ or $n^{-1} \mu(n) \rightarrow \infty$, we have proved in the theorem that the asymptotic value of number of oscillations of $P_{n}(t)$ about $y=K=\sqrt{2^{n} e^{\mu(n)}}$ is less than $\sqrt{n} / 2$. If $\mu(n)=O(n)$ the number of oscillations continues to be a fraction of $\sqrt{n}$ and decreases with the increase in value of $\mu(n)$. If $n^{-1} \mu(n) \rightarrow \infty$ the number of oscillations is reduced to $o_{p}(\sqrt{n})$. Inequality (35) together with Lemma 14 provides a glimpse of the manner in which the number of oscillations decreases with increase in value of $K$. Inequality (35) also shows that there is hardly any trace of the curve $P_{n}(t)$ above the level $y=K=\sqrt{2^{n} e^{\mu(n)}}$ if $\mu(n) /(n \log n) \rightarrow \infty$.

Let the coefficients $\left\{a_{k}(\omega)\right\}_{k=1}^{k=n}$ of $P_{n}(t)$ be standard normal random variables. The expected number of oscillations of $P_{n}(t)$ about the line $y=K, t \in(\alpha, \beta)$, has been denoted by us as $\operatorname{EN}_{n, K}(\alpha, \beta)$ in the following two theorems.

Theorem 1. For sufficiently large n,

$$
\mathrm{EN}_{n, 0}(-\infty, \infty)=\frac{\sqrt{n}}{2}+O_{p}\left(n^{-1 / 2} \log n\right)
$$

Theorem 2. For sufficiently large n,

(i) $\mathrm{EN}_{n, K}(-\infty, \infty)=\sqrt{n} / 2+O_{p}\left(n^{-1 / 2} \log n\right)$ if $K^{2} / 2^{n} \rightarrow$ $O$,

(ii) $\mathrm{EN}_{n, K}(-\infty, \infty)=\sqrt{n} / 2+(2 / \sqrt{\pi}) \operatorname{erf}\left(K / \sqrt{2^{n}}\right)+$ $O_{p}\left(n^{-1 / 2} \log n\right)$ if $K^{2}=O\left(2^{n}\right)$,

(iii) $\mathrm{EN}_{n, K}(-\infty, \infty)=\sqrt{n} / 2-n^{-1 / 2} \mu(n) /(2 \pi)+2 / \sqrt{\pi}+$ $O_{p}\left(n^{-1 / 2} \log n\right)$ if $\mu(n) / n \rightarrow 0, \mu(n) \rightarrow \infty$, and $K=$ $\sqrt{2^{n} e^{\mu(n)}}$

(iv) $\mathrm{EN}_{n, K}(-\infty, \infty)=(\sqrt{n} / \pi) \sin ^{-1}\left(e^{-n^{-1} \mu(n)} / \sqrt{2}\right)+$ $2 / \sqrt{\pi}+O_{p}\left(n^{-1 / 2} \log n\right)$ if $\mu(n)=O(n)$ and $K=$ $\sqrt{2^{n} e^{\mu(n)}}$ (v) $\mathrm{EN}_{n, K}(-\infty, \infty)=\sqrt{n} e^{-n^{-1} \mu(n)} /(2 \sqrt{2})+2 / \sqrt{\pi}+$ $O_{p}\left(n^{-1 / 2} \log n\right)$ if $\mu(n) / n \rightarrow \infty$ and $K=\sqrt{2^{n} e^{\mu(n)}}$.

Two more differences in behaviour of $P_{n}(t)$ and $Q_{n}(t)$ are noteworthy. In what follows we will find out that most of the axis crossings of $P_{n}(t)$ reach the level $\sqrt{2^{n} e^{\mu(n)}}$. However, the branches of $Q_{n}(t)$ that cross the axis do not travel beyond $y=$ $K$, where $K=O(\sqrt{n})$ (see Mahanti and Sahoo [6]). Almost all of the polynomial $Q_{n}(t)$ lie below the level $K$, where $n^{-1} \log (K / \sqrt{n}) \rightarrow \infty$ (Mahanti and Sahoo [6]). However, a large part of $P_{n}(t)$ stretches above this level.

\section{Formula for the Proof of the Theorems}

The proof of the theorem is based on the formula for expected number of level crossings given by Crammer and Leadbetter [9, page 285]. Using it for $P_{n}(t)-K=0$ in the interval $(\alpha, \beta)$ we can show that

$$
\begin{gathered}
\operatorname{EN}_{n, K}(\alpha, \beta)=\int_{\alpha}^{\beta} \frac{\sqrt{A_{n} C_{n}-B_{n}^{2}}}{A_{n}} \phi\left(-\frac{K}{\sqrt{A_{n}}}\right) \\
\cdot[2 \phi(\eta)+\eta\{2 \Phi(\eta)-1\}] d t,
\end{gathered}
$$

where $A_{n}=\operatorname{var}\left\{P_{n}(t)-K\right\}, B_{n}=\operatorname{cov}\left[\left\{P_{n}(t)-K\right\}, P_{n}^{\prime}(t)\right], C_{n}=$ $\operatorname{var}\left\{P_{n}^{\prime}(t)\right\}, \Phi(t)=(1 / \sqrt{2 \pi}) \int_{-\infty}^{t} \exp \left(-x^{2} / 2\right) d x, \phi(t)=$ $(1 / \sqrt{2 \pi}) \exp \left(-x^{2} / 2\right)$, and $\eta=-B_{n} K / \sqrt{A_{n}} \sqrt{A_{n} C_{n}-B_{n}^{2}}$. Since the coefficients of $P_{n}(t)$ are independent and $\operatorname{var}\left(a_{j}\right)=$ $\sqrt{\left(\begin{array}{l}n \\ k\end{array}\right)}$, it is easy to derive using little algebra that

$$
\begin{aligned}
A_{n} & =\sum_{k=1}^{n} \sqrt{\left(\begin{array}{l}
n \\
k
\end{array}\right)} \cosh ^{2} k t=2^{n-1}\left(\cosh n t \cosh ^{n} t+1\right), \\
B_{n} & =\frac{1}{2} \sum_{k=1}^{n} k \sqrt{\left(\begin{array}{l}
n \\
k
\end{array}\right)} \sinh 2 k t=2^{n-2} n \sinh (n+1) t \\
& \cdot \cosh ^{n-1} t, \\
C_{n} & =\sum_{k=1}^{n} k^{2} \sqrt{\left(\begin{array}{l}
n \\
k
\end{array}\right)} \sinh ^{2} k \\
& =2^{n-3}\left[\left(n^{2} \cosh (n+2) t+n \cosh n t\right) \cosh ^{n-2} t\right. \\
& \left.-n^{2}-n\right] .
\end{aligned}
$$

Let

$$
\begin{aligned}
D_{n} & =A_{n} C_{n}-B_{n}^{2}=2^{2 n-4}\left(n^{2}\left(\cosh ^{2 n} t-1\right)\right. \\
& +\left(n^{2}-n\right) \cosh ^{n-2} t \cosh n t \sinh ^{2} t \\
& +2 n^{2} \cosh ^{n-1} t \sinh n t \sinh t \\
& \left.+n\left(\cosh ^{2 n-2} t \cosh ^{2} n t-1\right)\right) .
\end{aligned}
$$


Formula (4) now can be written as

$$
\mathrm{EN}_{n, K}(\alpha, \beta)=I_{1}(\alpha, \beta)+I_{2}(\alpha, \beta)
$$

where

$$
\begin{aligned}
& I_{1}(\alpha, \beta)=\frac{1}{\pi} \int_{\alpha}^{\beta} \phi_{n}(t) \exp \left\{-\frac{K^{2} C_{n}}{2 D_{n}}\right\} d t, \\
& \quad=\int_{\alpha}^{\beta} \frac{\sqrt{2} K B_{n}}{\pi A_{n}^{3 / 2}} \exp \left\{-\frac{K^{2}}{2 A_{n}}\right\} \operatorname{erf}\left(\frac{K B_{n}}{\sqrt{2 A_{n} D_{n}}}\right) d t, \\
& \phi_{n}(t)=\frac{\sqrt{D_{n}}}{A_{n}} .
\end{aligned}
$$

As a special case, we can also obtain the famous Kac-Rice formula [10] for expected number of zeros of $P_{n}(t)$ by putting $K=0$ in (9). Thus we have

$$
\mathrm{EN}_{n, 0}(\alpha, \beta)=\frac{1}{\pi} \int_{\alpha}^{\beta} \phi_{n}(t) d t
$$

\section{Preliminary Analysis}

To evaluate the integrals in (9) and (11) we need to find out the dominant terms of $A_{n}, B_{n}$, and $C_{n}$. The inequalities mentioned in Lemmas 4-7 will be helpful for the purpose. We first mention the following form of L'Hôpital's Rule which we use to derive some of the inequalities.

Lemma 3 (the monotonic form of L'Hôpital's Rule [11]). For $-\infty<a<b<\infty$, let $g, h:[a, b] \rightarrow R$ be continuous on $[a, b]$ and differentiable on $(a, b)$ with $h^{\prime}(t) \neq 0$ on $(a, b)$. If $g^{\prime}(t) / h^{\prime}(t)$ is increasing or decreasing on $(a, b)$, then so are $(g(t)-g(a)) /(h(t)-h(a))$ and $(g(t)-g(b)) /(h(t)-h(b))$. If $g^{\prime}(t) / h^{\prime}(t)$ is strictly monotone, then the monotonicity of the above two quotients is also strict.

Lemma 4. If $0 \leq t<\pi / 2$, then $e^{n t^{2} / 2-n t^{4} / 8} \leq(\cosh t)^{n} \leq e^{n t^{2} / 2}$. In particular in the interval $\left(0, n^{-1 / 2}\right)$

$$
\begin{aligned}
1+ & \frac{n \sinh ^{2} t}{2}+\frac{n(n-2) \sinh ^{4} t}{8}<(\cosh t)^{n} \\
& <1+\frac{n \sinh ^{2} t}{2}+\frac{n^{2} \sinh ^{4} t}{4} .
\end{aligned}
$$

Thus $(\cosh t)^{n}=1+O\left(n t^{2}\right)$ if $t=o\left(n^{-1 / 2}\right)$.

Proof. The series representation of $\tanh t$ is given by [12, page 42] $\tanh t=\sum_{k=1}^{\infty} s_{k} t^{2 k-1}$, where $s_{k}=2^{2 k}\left(4^{k}-1\right) B_{2 k} /(2 k) !$. Using the fact that $\zeta(2 k)=2^{2 k-1} \pi^{2 k}\left|B_{2 k}\right| /(2 k)$ ! [12, page 1038], we have $\left|s_{k}\right| t^{2 k-1}=\pi^{-2 k}(2 t)^{2 k-1}\left(1-2^{-2 k}\right) \zeta(2 k)$.

Now, $\left(1-2^{-n}\right) \zeta(n)=\sum_{j=0}^{\infty}(2 j+1)^{-n}$ is a monotonically decreasing function of $n$. Therefore, the power series of tanh $t$ converges absolutely and uniformly for $0 \leq t<$ $\pi / 2$. Integrating term by term we have the alternative series $\log \cosh t=\sum_{k=1}^{\infty}(2 k)^{-1} s_{k} t^{2 k}$ for $0 \leq t<\pi / 2$. Since the coefficients of $t^{2 k}$ are monotonically decreasing, we find that the first part of the lemma is true.

Let $h_{1}(t)=r_{1}(t) / r_{2}(t)$, where $r_{1}(t)=(\cosh t)^{n}-1$ and $r_{2}(t)=n \sinh ^{2} t / 2+n^{2} \sinh ^{4} t / 4$, with $r_{1}(0)=r_{2}(0)=0$. Then

$$
\begin{gathered}
\left(\frac{r_{1}^{\prime}(t)}{r_{2}^{\prime}(t)}\right)^{\prime}=\left[(n-2)\left(n^{2}-4 n\right)(\cosh t)^{n-3} \sinh t\right. \\
\left.\cdot\left\{\sinh ^{2} t-\frac{(n+2)}{\left(n^{2}-4 n\right)}\right\}\right]\left\{2\left(1+n \sinh ^{2} t\right)^{2}\right\}^{-1} .
\end{gathered}
$$

It is easy to verify that $t+t^{3} / 5<(n+2) /\left(n^{2}-4 n\right)$ if $t \leq n^{-1 / 2}$. Using the series representation of $\sinh t$ we find that

$$
\begin{aligned}
t+\frac{t^{3}}{5}-\sinh t & =\frac{t^{3}}{30}-\sum_{k=2}^{\infty} \frac{t^{2 k+1}}{(2 k+1) !} \\
& \geq t^{3}\left(\frac{1}{30}-\sum_{k=2}^{\infty} \frac{1}{(2 k+1) !}\right) \geq 0 .
\end{aligned}
$$

Therefore $\sinh ^{2} t-(n+2) /\left(n^{2}-4 n\right)<0$ if $t \in\left(0, n^{-1 / 2}\right)$. As a consequence, $r_{1}^{\prime}(t) / r_{2}^{\prime}(t)$ is strictly decreasing in the interval $\left(0, n^{-1 / 2}\right)$. By Lemma $3, h_{1}(t)$ is decreasing in $\left(0, n^{-1 / 2}\right)$ and by L'Hôpital's Rule $h_{1}(0+)=1$. Therefore, $r_{1}(t)<r_{2}(t)$. Let $h_{2}(t)=r_{3}(t) / r_{4}(t)$, where $r_{3}(t)=(\cosh t)^{n}-1-\left(n \sinh ^{2} t\right) / 2$ and $r_{4}(t)=\left(n^{2} \sinh ^{4} t\right) / 4$ with $r_{3}(0)=r_{4}(0)=0$. Then $r_{3}^{\prime}(t) / r_{4}^{\prime}(t)=\left(\cosh ^{n-2} t-1\right)\left(n \sinh ^{2} t\right)^{-1}=r_{5}(t) / r_{6}(t)$, where $r_{5}(t)=(\cosh t)^{n-2}-1$ and $r_{6}(t)=n \sinh ^{2} t$ with $r_{5}(0)=$ $r_{6}(0)=0$. Now $r_{5}^{\prime}(t) / r_{6}^{\prime}(t)=(n-2)(\cosh t)^{n-4} /(2 n)$, which is increasing. So, by Lemma $3, h_{2}(t)$ is strictly increasing. By L'Hôpital's Rule we find that $h_{2}(0+)=(n-2) /(2 n)$. Therefore,

$$
1+\frac{n \sinh ^{2} t}{2}+\frac{n(n-2) \sinh ^{4} t}{8}<(\cosh t)^{n}
$$

Lemma 5. If $t \rightarrow 0$, then $\pi / 4+t / 2-t^{3} / 12<\tan ^{-1} e^{t}<$ $\pi / 4+t / 2-t^{3} / 12+t^{5} / 48$.

Proof. Let $r_{7}(t)=\tanh ^{-1} e^{t}-\pi / 4, r_{8}(t)=t / 2-t^{3} / 12$, and $r_{9}(t)=t / 2-t^{3} / 12+t^{5} / 48$. Observe that $r_{7}(0)=r_{8}(0)=$ $r_{9}(0)=0$. Let $h_{3}(t)=r_{7}(t) / r_{8}(t)$ and $h_{4}(t)=r_{7}(t) / r_{9}(t)$. Then $r_{7}^{\prime}(t) / r_{8}^{\prime}(t)=$ sec $h t$ is a monotonically decreasing and $r_{7}^{\prime}(t) / r_{9}^{\prime}(t)=2 \mathrm{sec} h t /\left(2-t^{2}\right)$ is monotonically increasing function of $t$. By L'Hôpital Rule $h_{3}(0+)=h_{4}(0+)=1$. Therefore, by Lemma 3, $r_{7}(t)<r_{8}(t)$ and $r_{9}(t)<r_{7}(t)$ for $t>0$.

Lemma 6. Let $m \geq 0, p \geq 1$. Then $\sinh ^{p} t(\sinh n t)^{-1}(\cosh t)^{-m}$ is a monotonically decreasing function of $t$ in $(0, \infty)$ if $p=1$ and is a monotonically decreasing function of $t$ in $(p / n, \infty)$ if $p \geq 2$ and $n>\sqrt{p}\{3(1-\tanh p)\}^{-1 / 2}$. 
Proof. Since

$$
\begin{aligned}
& \left(\sinh ^{p} t(\sinh n t)^{-1}(\operatorname{coth} t)^{-m}\right)^{\prime}=\sinh ^{p-1} t \\
& \cdot(\cosh t)^{m+1} \cosh n t \\
& \cdot\left(p \tanh n t-n \tanh t-m \tanh n t \tanh ^{2} t\right),
\end{aligned}
$$

to prove the lemma we show that $p \tanh n t-n \tanh t<0$ under the conditions mentioned in the lemma. We first note that $(\tanh n t-n \tanh t)^{\prime}=n\left(\sec h^{2} n t-\sec h^{2} t\right)<0$. Therefore, if $p=1$ and $t>0, p \tanh n t-n \tanh t<0$ since its value is zero at $t=0$.

Now let $p \geq 2$. Let $q(t)=r_{1}(t) / r_{2}(t)$, where $r_{1}(t)=$ $\tanh t$ and $r_{2}(t)=t$ with $r_{1}(0)=0, r_{2}(0)=0$. As $r_{1}^{\prime}(t) / r_{2}^{\prime}(t)=\sec ^{2} t$ is a decreasing function of $t$, it follows from Lemma 3 that $t^{-1} \tanh t$ is monotonically decreasing. Therefore, for a fixed $p,(p / n)^{-1} \tanh (p / n)$ increases with $n$. As $l t_{n \rightarrow \infty}(p / n)^{-1} \tanh (p / n)=1$ and $\tanh p<1$, there exists an integer $n_{0}$ such that, for $n>n_{0},(p / n)^{-1} \tanh (p / n)>\tanh p$. In other words, for $n>n_{0}, p \tanh n t-n \tanh t<0$ at $t=p / n$. Since $(\tanh n t-n \tanh t)^{\prime}<0$, we conclude that $p \tanh n t-n \tanh t<0$ if $t \geq p / n$ and $n>n_{0}$. We can find out $n_{0}$ in the following manner.

We note that $t^{-1} \tanh t-1+t^{2} / 3>0$ as $\left(\tanh t-t+t^{3} / 3\right)^{\prime}=$ $t^{2} \operatorname{sech}^{2} t\left(\cosh ^{2} t-t^{-2} \sinh ^{2} t\right)>0$ and tanh $t-t+t^{3} / 3$ is zero at $t=0$. So $(p / n)^{-1} \tanh (p / n)>\tanh p$ if $1-p^{2} /\left(3 n^{2}\right)>\tanh p$; that is, $n>n_{0}=\sqrt{p}\{3(1-\tanh p)\}^{-1 / 2}$.

Lemma 7. If $0 \leq x \leq 1 / \sqrt{2}$, then

$$
\begin{gathered}
\frac{(\pi / 2) x \sqrt{1-x^{2}}}{\left(1+2^{-1}\left(1-2 x^{2}\right)\right)^{1.27 \ldots}}<\sin ^{-1} x \\
<\frac{(\pi / 2) x \sqrt{1-x^{2}}}{\left(1+2^{-1}\left(1-2 x^{2}\right)\right)^{1.1137 \ldots}} .
\end{gathered}
$$

Proof. Let $x=\sin \theta$ and $\alpha=2 \theta$. Let $r_{10}(\theta)=\log \left(1+2^{-1} \cos \alpha\right)$ and $r_{11}(\theta)=\log (\pi \sin \alpha /(2 \alpha))$. We observe that $r_{10}(\pi / 2)=$ $r_{11}(\pi / 2)=0$. After differentiation we have

$$
\left(\frac{r_{11}^{\prime}(\theta)}{r_{10}^{\prime}(\theta)}\right)^{\prime}=\left(\frac{(2+\cos \alpha)\left(\alpha^{-1}-\cot \alpha\right)}{\sin \alpha}\right)^{\prime}=\frac{2\left[(2+\cos \alpha)\left(\operatorname{cosec}^{2} \alpha-\alpha^{-2}\right) \sin \alpha+\left(\cot \alpha-\alpha^{-1}\right)(1+2 \cos \alpha)\right]}{\sin ^{2} \alpha}
$$

We now use the following power series representation (see [12]) convergent for $|t|<\pi^{2}, t^{-1}-\cot t=$ $\sum_{k=1}^{\infty}\left(2^{2 k}\left|B_{2 k}\right| /(2 k) !\right) t^{2 k-1}$, where $B_{2 k}$ is the Bernoulli number of degree $2 k$.

Then,

$$
\begin{aligned}
& \sin ^{2} \alpha\left(\frac{r_{11}^{\prime}(\theta)}{r_{10}^{\prime}(\theta)}\right)^{\prime}=2 \sum_{k=1}^{\infty} \frac{2^{2 k}\left|B_{2 k}\right|}{(2 k) !}(\alpha)^{2 k-2} \\
& \cdot((2 k-1)(2+\cos \alpha) \sin \alpha-\alpha(1+2 \cos \alpha)) .
\end{aligned}
$$

We observe that $(2+\cos \alpha) \sin 2 \theta-\alpha(1+2 \cos \alpha)$ is nonnegative in $(0, \pi / 4)$ since it vanishes at zero and its derivative, that is, $2\left(\alpha \sin \alpha-\sin ^{2} \alpha\right)$ is positive.

It follows from (19) that $\left(r_{11}^{\prime}(\theta) / r_{10}^{\prime}(\theta)\right.$ is a nondecreasing function in $(0, \pi / 4)$ since

$$
\begin{gathered}
(2 k-1)(2+\cos \alpha) \sin \alpha-\alpha(1+2 \cos \alpha) \\
\geq(2+\cos \alpha) \sin 2 \theta-\alpha(1+2 \cos \alpha) .
\end{gathered}
$$

$l t_{\theta \rightarrow 0} r_{11}(\theta) / r_{10}(\theta)=\ln (\pi / 2) / \ln (3 / 2)=1.1137 \ldots$ and by L'Hôpital's Rule $l t_{\theta \rightarrow \pi / 4} r_{11}(\theta) / r_{10}(\theta)=4 / \pi=1.27 \ldots$, we obtain the proof of Lemma 7 using Lemma 3.

Lemma 8. Consider

$$
b_{0}-b_{1}<\frac{\sqrt{\pi}}{2} \int_{0}^{v} e^{-q^{2} x^{2}} \operatorname{erf}(x) d x<b_{0}
$$

where

$$
b_{k}=\frac{\left(1-e^{-(q v)^{2}} \sum_{j=0}^{k}\left((q v)^{2 j} / j !\right)\right)}{\left(2(2 k+1) q^{2 k+2}\right)}, \quad q>1 \text {. }
$$

Proof. Consider the following power series representation of $\operatorname{erf}(x)$ [12, eq. 3.321.1] $\operatorname{erf}(x)=(2 / \sqrt{\pi}) \sum_{k=0}^{k=\infty}(-1)^{k} x^{2 k+1} /$ $(k !(2 k+1))$. The power series converges absolutely and uniformly for $x \in R$. Hence [12, page 346]

$$
\begin{aligned}
& \frac{\sqrt{\pi}}{2} \int_{0}^{v} e^{-q^{2} x^{2}} \operatorname{erf}(x) d x \\
& =\sum_{k=0}^{k=\infty}(-1)^{k} \frac{\int_{0}^{v} x^{2 k+1} e^{-q^{2} x^{2}} d x}{k !(2 k+1)} \\
& =\sum_{k=0}^{k=\infty}(-1)^{k} \frac{\gamma\left(k+1, q^{2} v^{2}\right)}{2 q^{2 k+2} k !(2 k+1)}=\sum_{k=0}^{k=\infty}(-1)^{k} b_{k},
\end{aligned}
$$

where $b_{k}=\left(1-e^{-(q v)^{2}} \sum_{j=0}^{k}\left((q v)^{2 j} / j !\right)\right) /\left(2(2 k+1) q^{2 k+2}\right)$. It is easy to see that $b_{k}$ is nonincreasing and $b_{k} \rightarrow 0$ as $k \rightarrow \infty$. Thus, the statement of the lemma is true.

Lemma 9. Let $u_{n}=o\left(n^{-1 / 2}\right)$; then

$$
\int_{0}^{u_{n}} \phi_{n}(t) d t=O\left(u_{n} \sqrt{n}\right)
$$


Proof. By Lemma 4, $D_{n}$ defined in (8) can be written as

$$
\begin{aligned}
D_{n} & =2^{2 n-4} n \sinh ^{2} n t \\
& \cdot\left\{(n-1) \sinh ^{2} t \operatorname{cosech} n t\left(\cosh ^{2} n t-\cosh n t\right)\right. \\
& \left.+(n \sinh t \operatorname{cosech} n t+1)^{2}\right\}\left(1+O(n t)^{2}\right) .
\end{aligned}
$$

By Lemma 6 we find that $(n \sinh t \cos e c h n t+1)^{2}=O(1)$ in $(0,1)$ and $(\sinh t \operatorname{cosech} n t)^{2} \cosh ^{2} n t=O\left(n^{-1}\right)$ if $n t=O(1)$. Also, $\sinh ^{2} t \operatorname{coth}^{2} n t=O\left(t^{2}\right)$ if $n t \rightarrow \infty$ and $t \in(0,1)$. So, $\left(2^{2 n-4} n \sinh ^{2} n t\right)^{-1} D_{n} \sim C_{1}, \quad 1 \leq C_{1} \leq 2$. Also using Lemma 4 and (5) we find that $A_{n} / 2^{n-1} \sim \cosh n t+1$. By (11), we see that Lemma 9 is valid.

Lemma 10. If $\beta>\alpha \geq 2 n^{-1} \log n$, then

$$
\begin{aligned}
\int_{\alpha}^{\beta} \phi_{n}(t) d t= & \sqrt{n}\left(\tan ^{-1} e^{\beta}-\tan ^{-1} e^{\alpha}\right) \\
& +O\left(n^{3 / 2} \sinh \alpha \exp (-n \alpha)\right) .
\end{aligned}
$$

Proof. Using Lemmas 6 and 4 we obtain from (8) that $D_{n}=$ $2^{2 n-4} n \cosh ^{2 n-2} t \cosh ^{2} n t(1+O(n \sinh u \exp (-n u)))$ and $A_{n}=$ $2^{n-1} \cosh n t \cosh ^{n} t(1+O(\exp (-n u)))$. The conclusion of the lemma follows now from (11).

\section{Proof of Theorem 1}

If $u_{n}$ is taken as $2 n^{-1} \log n$ we can derive from Lemmas 9 and 10 the following relations:

$$
\begin{aligned}
& \mathrm{EN}_{n, 0}\left(0,2 n^{-1} \log n\right)=O_{p}\left(n^{-1 / 2} \log n\right), \\
& \mathrm{EN}_{n, 0}\left(2 n^{-1} \log n, \infty\right) \\
& \quad=\frac{\sqrt{n}}{2}-\frac{\sqrt{n} \tan ^{-1} e^{2 n^{-1} \log n}}{\pi}+O_{p}\left(n^{-1 / 2}\right) .
\end{aligned}
$$

Since the integrand in (11) is an even function of $t$, we have

$$
\mathrm{EN}_{n, 0}(-\infty, \infty)=2 \mathrm{EN}_{n, 0}(0, \infty) .
$$

Using Lemma 5 to approximate $\tan ^{-1} e^{2 n^{-1} \log n}$, we see that Theorem 1 is true.

\section{Proof of Theorem 2}

To determine $\operatorname{EN}_{n, K}(-\infty, \infty)$ we need to calculate $I_{1}(-\infty, \infty)$ and $I_{2}(-\infty, \infty)$ for different ranges of values of $K$ as both quantities depend on magnitude of $K$. In Lemma 14 we have calculated $I_{2}(-\infty, \infty)$. Value of $I_{1}(-\infty, \infty)$ for three different ranges of value of $K$ has been calculated in Lemmas 11-13. The relations (32), (34), and (35) and Lemma 14 establish Theorem 2 . Note that we only need to calculate $I_{1}(0, \infty)$ and $I_{2}(0, \infty)$ since $I_{1}(\alpha, \beta)$ and $I_{2}(\alpha, \beta)$ are even functions of $t$.

Since $I_{1}(\alpha, \beta) \leq \operatorname{EN}_{n, 0}(\alpha, \beta)$, by Lemma 9 we have

$$
I_{1}\left(0,2 n^{-1} \log n\right)=O_{p}\left(n^{-1 / 2} \log n\right) .
$$

In order to calculate $I_{1}\left(2 n^{-1} \log n, \infty\right)$, we need the dominant term of $C_{n} / D_{n}$, which can be calculated with the help of Lemmas 4 and 6 as

$$
\frac{C_{n}}{D_{n}}=n\left(2^{n-1} \cosh n t \cosh ^{n} t\right)^{-1}\left(1+O\left(n^{-1}\right)\right) .
$$

For brevity, we have written $K^{\prime}=K^{2} / 2^{n}$ in the following lemmas.

Lemma 11. As long as $K^{\prime}$ does not exceed $e^{\mu(n)}$, where $\mu(n)=$ $o(\sqrt{n})$,

$$
I_{1}(0, \infty)=\frac{\sqrt{n}}{4}+O_{p}\left(n^{-1 / 2} \log n\right)
$$

Proof. Let $K^{\prime} \leq n^{\alpha}, \alpha>0$. Then by Lemma 9 we find that $I_{1}\left(0, n^{-1}(\alpha+2) \log n\right)=O_{p}\left(n^{-1 / 2} \log n\right)$. Using (30) and Lemma 10, we obtain that $I_{1}\left(n^{-1}(\alpha+2) \log n, \infty\right)=\sqrt{n} / 4+$ $O_{p}\left(n^{-1 / 2} \log n\right)$. Hence $I_{1}(0, \infty)=\sqrt{n} / 4+O_{p}\left(n^{-1 / 2} \log n\right)$.

Now let $K^{\prime} \geq n^{\alpha}, \alpha \geq 2$, and $n^{-1 / 2} \log \left(K^{\prime}\right)=o(1)$. It follows from (30) that the maximum value of $e^{-K^{\prime} C_{n} D_{n}^{-1}}$ in $\left(2 n^{-1} \log n, n^{-1} \log \left(n K^{\prime 2} / \log n\right)\right)$ is $O\left(n^{-1}\right)$.

Hence $I_{1}\left(\left(2 n^{-1} \log n, n^{-1} \log \left(n K^{\prime 2} / \log n\right)\right)\right)=O_{p}\left(n^{-1 / 2}\right)$. As the integrand of $I_{1}(a, b)$ is bounded, we have $I_{1}\left(n^{-1} \log \left(n K^{\prime} / \log n\right), n^{-1} \log \left(K^{\prime 2} n^{2}\right)\right)=O_{p}\left(n^{-1 / 2}\right)$. By Lemma 10 and (30) we find that $I_{1}\left(n^{-1} \log \left(K^{\prime} n^{2}\right), \infty\right)=$ $\sqrt{n} / 4+O_{p}\left(n^{-1 / 2}\right)$. Taking into account (29) we see that (31) is true.

Lemma 12. Let $n^{-1 / 2} \log K^{\prime}=\mu(n)$, where either $\mu(n)=O(1)$ or $\mu(n) \rightarrow \infty$, but $\mu(n)=o(\sqrt{n})$; then

$$
I_{1}(0, \infty)=\frac{\sqrt{n}}{2}-\frac{\mu(n)}{2 \pi}+O_{p}\left(n^{-1 / 2} \log n\right)
$$

Proof. By Lemma 10 and (30) we find that the following relations are true:

$$
\begin{aligned}
& I_{1}\left(2 n^{-1} \log n,(2 n)^{-1} \log n+\mu(n) n^{-1 / 2}-\frac{\mu^{2}(n)}{2}\right) \\
& \quad=O_{p}\left(\sqrt{n} e^{-\sqrt{n}}\right), \\
& I_{1}\left(2 n^{-1} \log n+\mu(n) n^{-1 / 2}-\frac{\mu^{2}(n)}{2}, \infty\right)=\frac{\sqrt{n}}{4} \\
& \quad-\frac{\mu(n)}{\pi}+O_{p}\left(n^{-1 / 2}\right), \\
& I_{1}\left((2 n)^{-1} \log n+\mu(n) n^{-1 / 2}-\frac{\mu^{2}(n)}{2}, 2 n^{-1} \log n\right. \\
& \left.\quad+\mu(n) n^{-1 / 2}-\frac{\mu^{2}(n)}{2}\right)=O_{p}\left(n^{-1 / 2} \log n\right) .
\end{aligned}
$$

These relations and (29) yield (32). 
Lemma 13. Let $\log K^{\prime}=n \mu(n)$, where $\mu(n)=O(1)$ or $\mu(n) \rightarrow$ $\infty$. Then

$$
\begin{aligned}
I_{1}(0, \infty)= & \left(\frac{2 \sqrt{n}}{\pi}\right) \sin ^{-1}\left(\frac{e^{-\mu(n)}}{\sqrt{2}}\right) \\
& +O_{p}\left(n^{-1 / 2} \log n\right) .
\end{aligned}
$$

For large values of $n$

$$
\begin{gathered}
\frac{\sqrt{n} x \sqrt{1-x^{2}}}{4\left(1+\sqrt{1-x^{2}+x^{4}}\right)^{1.27 \ldots}}<I_{1}(0, \infty) \\
<\frac{\sqrt{n} x \sqrt{1-x^{2}}}{4\left(1+\sqrt{1-x^{2}+x^{4}}\right)^{1.1137 \ldots}},
\end{gathered}
$$

where $x=e^{-\mu(n)} / \sqrt{2}$.

Moreover, $I_{1}(0, \infty)=O_{p}(1)$ if $\mu(n)=O(n \log n)$ and $I_{1}(0, \infty)=o_{p}(1)$ if $\mu(n) /(n \log n) \rightarrow \infty$.

Proof. Let $t_{1}$ and $t_{2}$ be the points in $R$ where $(\exp (2 t)+1)^{n} / 2^{n}$ assumes the values $(n / \log n) \exp (n \mu(n))$ and $n^{2} \exp (n \mu(n))$, respectively. It follows from (30) and Lemma 10 that

$$
I_{1}\left(2 n^{-1} \log n, t_{1}\right)=O_{p}\left(n^{-1 / 2}\right) .
$$

Let $z=\ln \left(\left(e^{2 t}+1\right) / 2\right)$ and $s=2 e^{z}$.

Then $\int \sec h t d t=\int\left(d z / \sqrt{2 e^{z}-1}\right)=\int(d s / s \sqrt{s-1})=$ $-2 \sin ^{-1}\left(s^{-1 / 2}\right)$. Therefore, by (30) and the definition of $I_{1}(\alpha, \beta)$, we have $I_{1}\left(t_{1}, t_{2}\right)=O_{p}\left(n^{-1 / 2} \log n\right)$ and $I_{1}\left(t_{2}, \infty\right)=$ $\sqrt{n} \sin ^{-1}\left(e^{-\mu(n)} / \sqrt{2}\right)+O_{p}\left(n^{-1 / 2)}\right)$. We obtain (34) if (29) is also considered. Inequality (35) follows immediately from Lemma 7. From this inequality the other two estimates of $I_{1}(0, \infty)$ valid for $\mu(n)=O_{p}(n \log n)$ and $\mu(n)$ such that $\mu(n) /(n \log n) \rightarrow \infty$ follow.

Lemma 14. The dependence of $I_{2}(-\infty, \infty)$ on $K$ is given by the following relations:

(i) if $K^{2}=o\left(2^{n}\right)$, then $I_{2}(-\infty, \infty)=o_{p}(1)$,

(ii) if $K^{2}=O\left(2^{n}\right)$, then $I_{2}(-\infty, \infty)=(2 / \sqrt{\pi}) \operatorname{erf}(u)+$ $\mathrm{O}_{p}\left(e^{-n}\right)$,

(iii) if $K^{2} / 2^{n} \rightarrow \infty$, then $I_{2}(-\infty, \infty)=(2 / \sqrt{\pi})+$ $\mathrm{O}_{p}\left(e^{-K^{2} / 2^{n}}\right)$

where $u=\sqrt{K^{\prime}}$.

Proof. Using Lemma 4 we find that in $\left(0, u_{n}\right)$

$$
\begin{aligned}
& D_{n}=2^{2 n-4} n \sinh ^{2} n t(1+2 n \sinh t \operatorname{cosech} n t+o(1)), \\
& B_{n}=2^{n-2} n \sinh ^{2} n t(1+o(1)) .
\end{aligned}
$$

Hence, $B_{n} / \sqrt{D_{n}} \sim \sqrt{n} / c$, where $1 \leq c \leq \sqrt{3}$.
On the other hand, using Lemma 6 and the definitions of $B_{n}$ and $D_{n}$ in $\left(u_{n}, \infty\right)$, where $u_{n}>2 n^{-1} \log n$, we have $B_{n} / \sqrt{D_{n}} \sim \sqrt{n} e^{t}$.

Let $s=K / \sqrt{2 A_{n}}$. It is not difficult to see that $(K / s)^{1 / n} \sim e^{t}$ in the interval $\left(u_{n}, \infty\right)$

$$
\begin{aligned}
I_{2}(-\infty, \infty)= & 2 I_{2}(0, \infty) \\
= & \left(\frac{4}{\pi}\right) \int_{0}^{u} \exp \left(-s^{2}\right) \operatorname{erf}(s p) d s \\
= & \left(\frac{2}{\sqrt{\pi}}\right) \operatorname{erf}(u p) \operatorname{erf}(u) \\
& -4 p \int_{0}^{u} \exp \left(-p^{2} s^{2}\right) \operatorname{erf}(s) d s,
\end{aligned}
$$

where $\sqrt{n} / \sqrt{3} \leq p \leq \sqrt{n} e^{\mu(n)}$.

Clearly $I_{2}(-\infty, \infty)=o_{p}(1)$ if $K^{2}=o\left(2^{n}\right)$. The other two parts of the lemma are found to be true by virtue of Lemma 8 .

\section{Conflict of Interests}

The authors declare that there is no conflict of interests regarding the publication of this paper.

\section{References}

[1] K. Farahmand, Topics in Random Polynomials, vol. 393 of Pitman Research Notes in Mathematics Series, Longman, Harlow, UK, 1998.

[2] M. Das, On real zeros of random polynomial with hyperbolic elements [Ph.D. thesis], Utkal University, Bhubaneswar, India, 1971.

[3] K. Farahmand, "Level crossings of a random polynomial with hyperbolic elements," Proceedings of the American Mathematical Society, vol. 123, no. 6, pp. 1887-1892, 1995.

[4] M. K. Mahanti, "Expected number of real zeros of random hyperbolic polynomial," Statistics \& Probability Letters, vol. 70, no. 1, pp. 11-18, 2004.

[5] M. K. Mahanti, "On expected number of real zeros of a random hyperbolic polynomial with dependent coefficients," Applied Mathematics Letters, vol. 22, no. 8, pp. 1276-1280, 2009.

[6] M. K. Mahanti and L. Sahoo, "On expected number of level crossings of random hyperbolic polynomial," Rocky Mountain Journal of Mathematics. In press.

[7] J. R. Wilkins, "Mean number of real zeros of random hyperbolic polynomial," International Journal of Mathematics and Mathematical Sciences, vol. 23, no. 5, pp. 335-342, 2000.

[8] A. Edelman and E. Kostlan, "How many zeros of a random polynomial are real?" Bulletin of the American Mathematical Society, vol. 32, no. 1, pp. 1-37, 1995.

[9] H. Crammer and A. R. Leadbetter, Stationary and Related Stochastic Process, Wiley, New York, NY, USA, 1967.

[10] M. Kac and S. O. Rice, "Mathematical theory of random noise," Bell System Technical Journal, 1945, Reprinted in: Selected Papers Noise and Stochastic Processes, pp. 133-294, Dover, NY, USA, edited by: N. Wax, 1954. 
[11] G. D. Anderson, M. K. Vamanamurthy, and M. K. Vuorinen, Conformal Invariants, Inequalities and Quasiconformal Maps, Canadian Mathematical Society Series of Monographs and Advanced Texts, John Wiley \& Sons, New York, NY, USA, 1997.

[12] I. S. Gradshteyn and I. M. Ryzhik, Tables of Integrals, Series and Products, Academic Press, New York, NY, USA, 7th edition, 2007. 


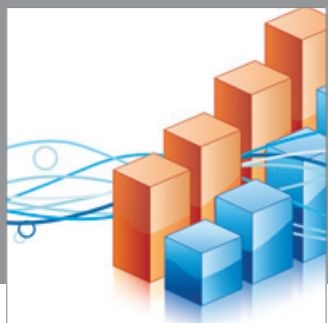

Advances in

Operations Research

mansans

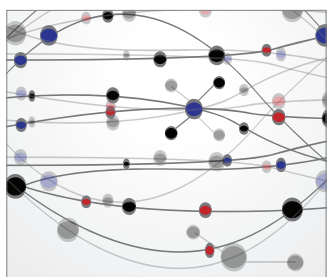

The Scientific World Journal
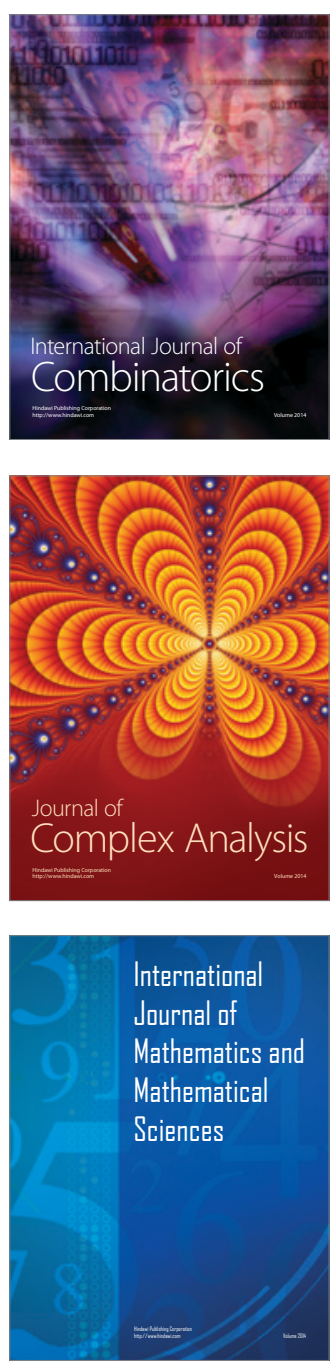
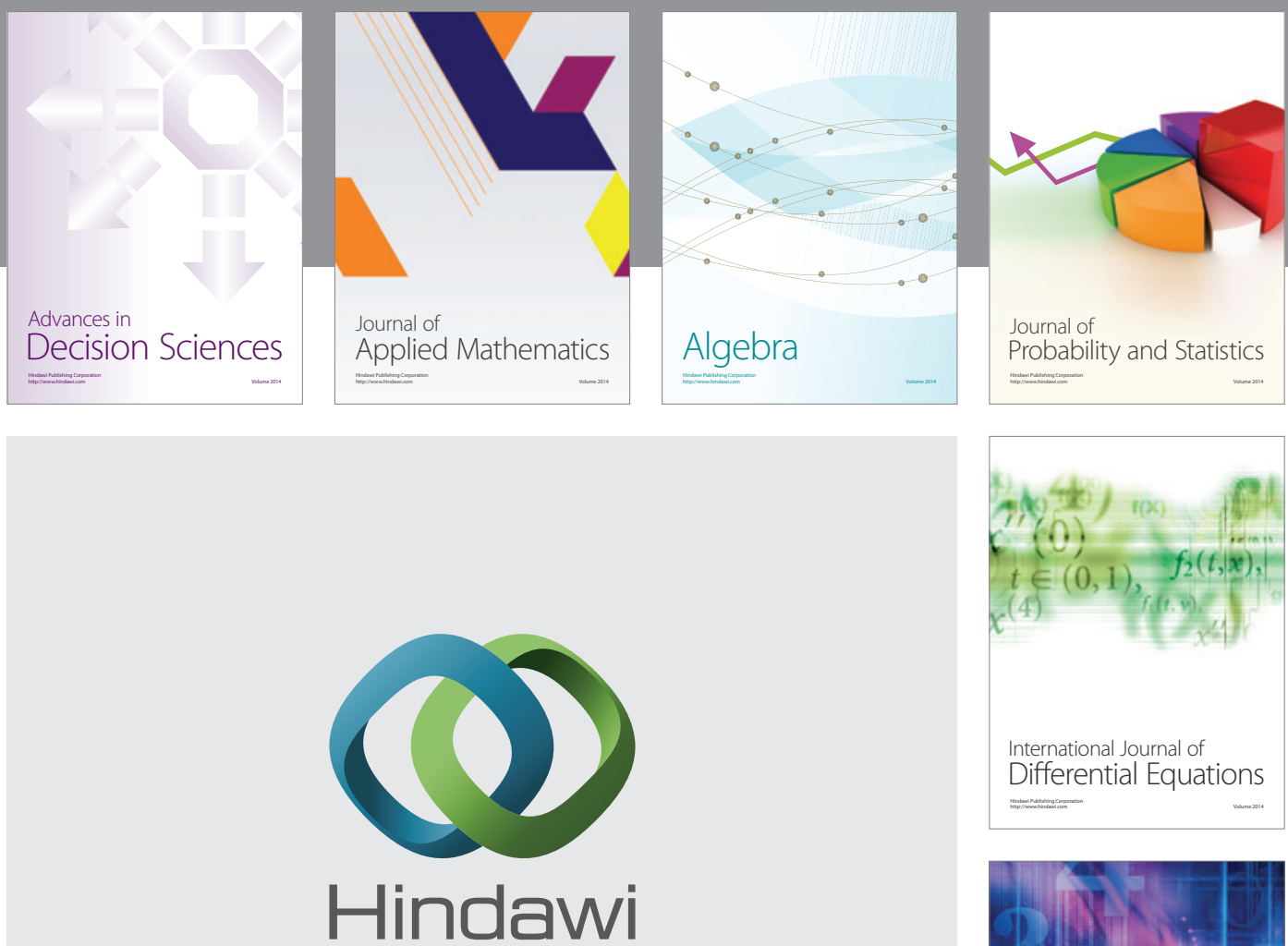

Submit your manuscripts at http://www.hindawi.com
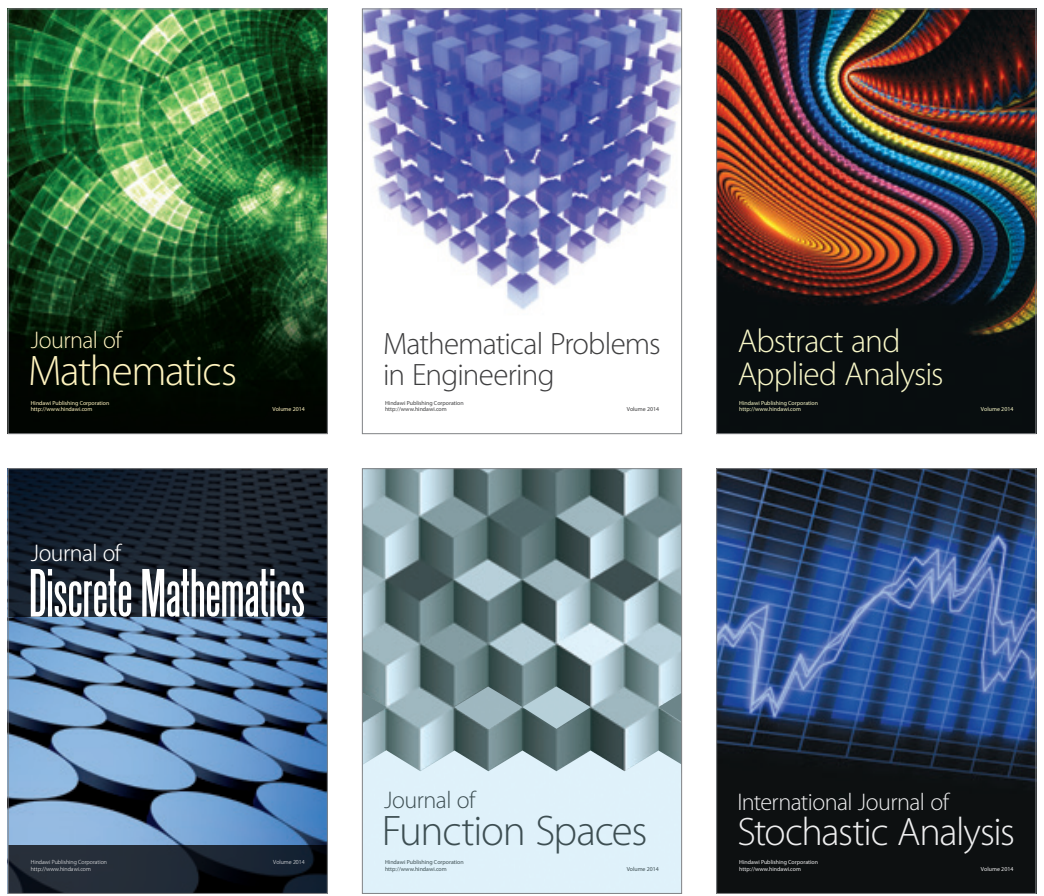

Journal of

Function Spaces

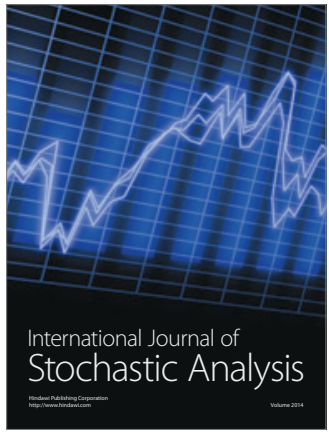

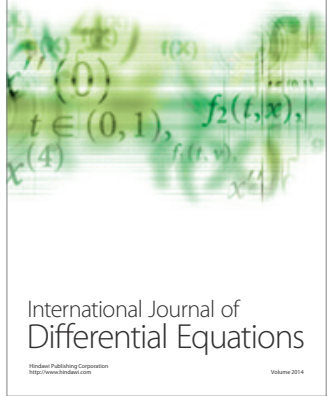
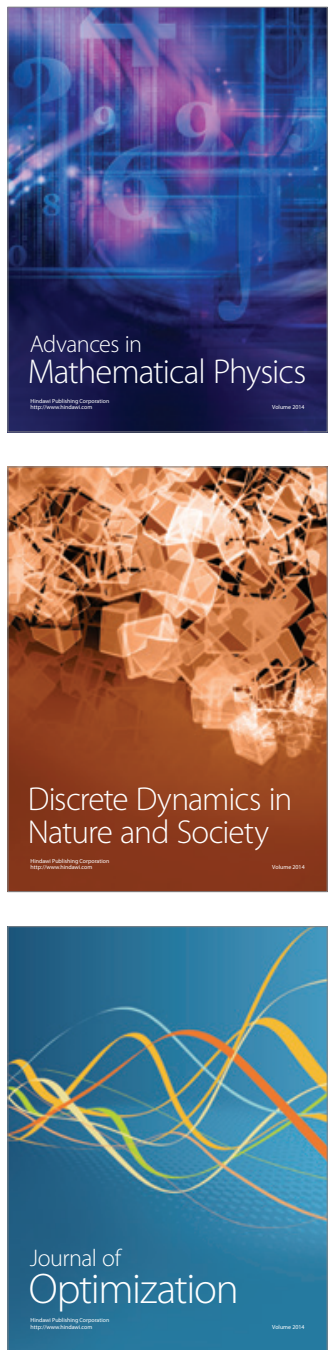